\section{El aprendizaje basado en problemas. De herejía artificial a res popularis}

\author{
L.A. Branda
}

Cuestionario

de acreditación

www.educmed.net
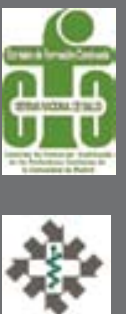

La extensa implementación del aprendizaje basado en problemas (ABP) en el proceso enseñanza-aprendizaje ha resultado en su transformación de herejía artificial a res popularis con la consecuente proliferación de publicaciones, libros y congresos sobre el tema. A menudo, esta avalancha de información, ha creado una confusión en la comprensión de qué es el ABP como estrategia de aprendizaje. Este artículo presenta al lector una definición de lo que se consideró que era el ABP y su extensión, además de incluir la resolución de problemas. Se indica la importancia de los objetivos de aprendizaje (resultados del aprendizaje) y se presentan algunos pasos que se deben seguir en la preparación de situaciones/ escenarios/problemas/casos. De forma general, se describen la evaluación de los estudiantes fundamentalmente formativa, basada en las observaciones hechas en las sesiones de tutoría, y la evaluación de carácter sumativo. La descripción de las etapas más comunes en el ABP tiene el propósito de indicar lo que los estudiantes pueden hacer y no que deben hacer. Si se consideran las limitaciones de recursos que tienen la mayoría de las instituciones que desean implementar el ABP, se describe la aplicación de esta estrategia en grupos grandes. Se discute el rol del tutor facilitador y se indican las características de sus intervenciones en un continuo que va desde jerárquica a facilitadora de la autonomía del estudiante en su aprendizaje. Este artículo finaliza con una reflexión sobre el aprendizaje autodirigido y su relación con el aprendizaje autorregulado.

Palabras clave. Aprendizaje autodirigido. Aprendizaje basado en problemas. Evaluación. Grupos grandes. Resolución de problemas.

\section{Problem based learning. From artificial heresy to res popularis}

The vast use of problem based learning (PBL) in the teachinglearning process has resulted in its transformation from an artificial heresy to a res popularis with the subsequent proliferation of publications, books and congresses on the subject. This deluge of information, very often, has created confusion on the comprehension of what PBL is as learning strategy. This article presents a definition of what PBL was considered in its conception, and its extension to problem resolution. The importance of learning objectives (learning outcomes) is indicated and some steps in the preparation of situations/scenarios/problems/cases are described. Also, student evaluation is described, both the formative evaluation based on tutorial observations as well as the summative. The description of the stages in the PBL process has solely the purpose to indicate what the students could do, not what they should do. Taking into account the resource limitations of most institutions which wish to implement PBL, the application of this strategy in large groups is also described. The role of the tutor facilitator and the characteristics of his intervention from hierarchical to one of facilitating student autonomy are discussed. This article ends with a reflexion on self-directed learning and its relationship to self-regulated learning.

Key words. Evaluation. Large groups. Problem based learning. Problem solving. Self-directed learning.

\section{Reflexiones iniciales con palabras de cautela}

Los cambios en la educación médica, sean innovaciones o modificaciones de ruta y estrategias, 
tradicionalmente han sido difíciles de aceptar. El moralista francés Jean des Caurres reaccionó airadamente a la innovación que fue introducir en el siglo xvi el espejo de vidrio el cual sustituyó al de metal bruñido ('O Dieu! Hélas, en que mal heureux regne sommes-nous tombés? Oevres morales et Divers'). Implícita y, a menudo explícitamente, cuando al final de la década de 1960 la Universidad de McMaster introdujo el aprendizaje basado en problemas (ABP) en el estudio de medicina, la reacción en el ámbito de la educación médica fue similar: ‘¡Ay, bajo qué perversas influencias hemos caído! La introducción del ABP en el aprendizaje de medicina fue recibida por el entorno educacional dominante en medicina con una actitud de tolerante curiosidad, en el mejor de los casos. Malcom Lambert, en su libro La herejía medieval, desarrolla el concepto de 'herejía artificial' para referirse a las excusas que el celo inquisitorial utilizó para perseguir la heterodoxia -real o percibida-. Durante un tiempo la heterodoxia, que constituía el ABP, fue ignorada e incluso sufrió el acoso de la inquisición reinante en la educación médica que resistía cualquier cambio fundamental en la enseñanza. Casi 40 años han pasado desde que el ABP fue introducido en medicina, lo cual ahora es considerado un hito en educación médica, y ha alcanzado la dudosa distinción de ser popular en el ámbito de la pedagogía. Esta situación de res popularis del $\mathrm{ABP}$ ha resultado en una miríada de publicaciones, tanto en revistas como en libros. Algunas de las publicaciones que relatan la implementación del ABP han sido meritorias, puesto que describen su adaptación al entorno donde esta estrategia se aplica y han contribuido de forma significativa en la bibliografía de cómo esta estrategia es aplicable de manera diversa y, en algunos casos, con demostrada efectividad para el aprendizaje del estudiante $[1,2]$. Sin embargo, la calidad de las publicaciones ha sido variable debido, quizás, a que se ha escrito mucho sobre un mismo tema. Algunas de estas publicaciones están plagadas de errores tanto históricos como conceptuales. Los errores históricos podrían explicarse por la falta de esmero en la revisión bibliográfica sobre el tema; los conceptuales podrían ser el resultado de la experiencia limitada de algunos de los autores en la implementación de esta estrategia. Esta profusión de publicaciones sólo se equipara a la epidemia de reuniones, conferencias, con- gresos y simposios sobre el ABP. Los asistentes a estos congresos pueden ser comparados con los peregrinos de la Edad Media, que disfrutan del placer de viajar al mismo tiempo que dan la impresión de estar dedicados a la reflexión y al aprendizaje. Se ha dicho que los ejercicios de penitencia de este peregrinaje son las presentaciones de comunicaciones -generalmente recicladas- y oír las comunicaciones de otros, también recicladas. Sin duda estas reuniones tienen el mérito de dar la oportunidad a los congresistas de conocer personas interesantes que forman parte de la intelectualidad pedagógica reinante con las que establecen vínculos para formar redes virtuales futuras o reales sobre el ABP y regresar a casa dando la impresión de ser una persona consumada.

\section{¿En qué consiste el $A B P$ ?}

Pasar de ser una herejía artificial a ser una res popularis ha oscurecido la comprensión de lo que es realmente esencial en el ABP como estrategia de aprendizaje. En los orígenes del ABP deben reconocerse sus antecesores conceptuales y aquellos que son antecedentes metodológicos. Un elemento del ABP que aún se considera el centro de esta estrategia es el aprendizaje autodirigido. Éste se puede trazar en sus orígenes a las Analectas de Confucio y, más posteriormente, a Wolfang Ratke, que en el siglo XVII insistía en que la autodisciplina del estudiante se debe llevar a cabo sin la interferencia de los profesores. Amos Comenius, de la misma época que Ratke, también insistía en el aprendizaje autodirigido y en que los maestros deben enseñar menos y los alumnos aprender más. Los métodos utilizados por Comenius también pueden considerarse precursores del ABP, desde que él utilizaba grupos de imágenes como generadores del aprendizaje del latín en la lengua vernácula. En la época contemporánea, un precursor del ABP puede considerarse ser el Functional Context Method of Instruction utilizado por Harry A. Shoemaker en 1960. Shoemaker enseñaba a sus estudiantes electrónica básica a través del proceso de reparación y mantenimiento de equipos de radio. Conceptualmente, el ABP puede enmarcarse dentro de las conocidas ideas promovidas por John Dewey y las del constructivismo. 


\section{Programa de Formación Médica Acreditada en Educación Médica}

Artículo: El aprendizaje basado en problemas: de herejía artificial a res popularis

Autor: L.A. Branda

Revista: Educ Med 2009; 12: 11-23

1. Si el punto de partida es una situación problemática, en el ABP se espera que el estudiante:

$\square$ a) Identifique que debe aprender

$\square$ b) Evalúe lo aprendido

$\square$ c) Identifique los objetivos de aprendizaje cumplidos

$\square$ d) Identifique los principios en los que se basa el conocimiento adquirido

$\square$ e) Haga todos los pasos previos

2. Si los objetivos de aprendizaje incluyen la resolución de problemas dada una situación problemática, se espera que el estudiante:

$\square$ a) Identifique cuál o cuales son los problemas

$\square$ b) Identifique lo que debe aprender para resolver el o los problemas

$\square$ c) Describa un plan de intervención e incluya barreras y recursos necesarios

$\square$ d) Evalúe la efectividad de la intervención

$\square$ e) Haga todos los pasos previos

3. En una asignatura/módulo/unidad de aprendizaje los objetivos de aprendizaje (resultados del aprendizaje) son expresados claramente por:

(indique todos los que considera apropiados)

$\square$ a) El contenido

$\square$ b) La lista de los temas

$\square$ c) La descripción en términos explícitos y sin ambigüedades de lo que se espera del estudiante

$\square$ d) La actividad que el estudiante debe llevar a cabo

$\square$ e) El contenido que ha de demostrar para ser satisfactorio

4. En el $A B P$, los pasos que se consideran útiles para la preparación de problemas son:

(indique todos los que considera apropiados)

$\square$ a) Construir una lista de situaciones posibles

$\square$ b) Adjudicar un valor educacional basado en los objetivos de aprendizaje

$\square$ c) Adjudicar una frecuencia a cada situación

$\square$ d) Identificar el posible impacto de cada situación

$\square$ e) Seleccionar del orden prioritario cuál se escribirá

5. En la evaluación del estudiante, resultante de las observaciones de tutorías del $A B P$, se debe tener en cuenta:

$\square$ a) La autoevaluación

$\square$ b) La coevaluación (evaluación por los pares)

$\square$ c) La evaluación del tutor facilitador

$\square$ d) Todo lo previo

$\square$ e) Nada de lo previo
6. En la evaluación sumativa, dada una situación problemática, se espera que el estudiante: (indique todos los que considera apropiados)

$\square$ a) Identifique el conocimiento que desea adquirir

$\square$ b) Seleccione las áreas de mayor importancia para el problema

$\square$ c) Lleve a cabo una búsqueda efectiva con un análisis crítico de la información recogida

$\square$ d) Demuestre comprensión del conocimiento adquirido

$\square$ e) Aplique el conocimiento adquirido a la situación problemática

7.Las etapasmás comunesenel ABPengrupospequeñosson: (indique todas las que considera apropiadas)

$\square$ a) Lluvia de ideas y confección de un plan de aprendizaje

$\square$ b) Búsqueda y análisis del conocimiento encontrado

$\square$ c) Discusión del conocimiento adquirido

$\square$ d) Resumen del conocimiento y abstracción

$\square$ e) Aplicación del conocimiento al problema en discusión

8. Las preguntas más comunes en la evolución de un grupo de tutoría de $A B P$ son:

$\square$ a) ¿Qué se supone que se debe hacer?

$\square$ b) ¿Se aprende lo suficiente?

$\square$ c) ¿Se aprende algo útil?

$\square$ d) ¿Se ha estabilizado el proceso de aprendizaje?

$\square$ e) Todo lo previo

9. En la aplicación del ABP en grupos grandes, después de la presentación de la situación problemática, las actividades siguientes son las más comunes:

(seleccione cuantas considere apropiadas):

$\square$ a) Clase expositiva sobre el tema relacionado con el problema

$\square$ b) Trabajo en grupos pequeños identificando lo que se desea aprender

$\square$ c) Clase magistral posterior a la identificación de lo que se desea aprender

$\square$ d) Sesión de aclaraciones de dudas por el docente

$\square$ e) Trabajo en grupos para identificar áreas que necesitan estudio posterior

10. Las intervenciones del tutor facilitador durante las sesiones de tutoría pueden tener las siguientes características: (indique todas las que considera apropiadas)

$\square$ a) Dirige

$\square$ b) Informa

$\square$ c) Confronta

$\square$ d) Desafía

$\square$ e) Comparte

Nombre y apellidos.

DNI.

Dirección .

Código postal

Localidad

Provincia/país

Teléfono E-mail. 


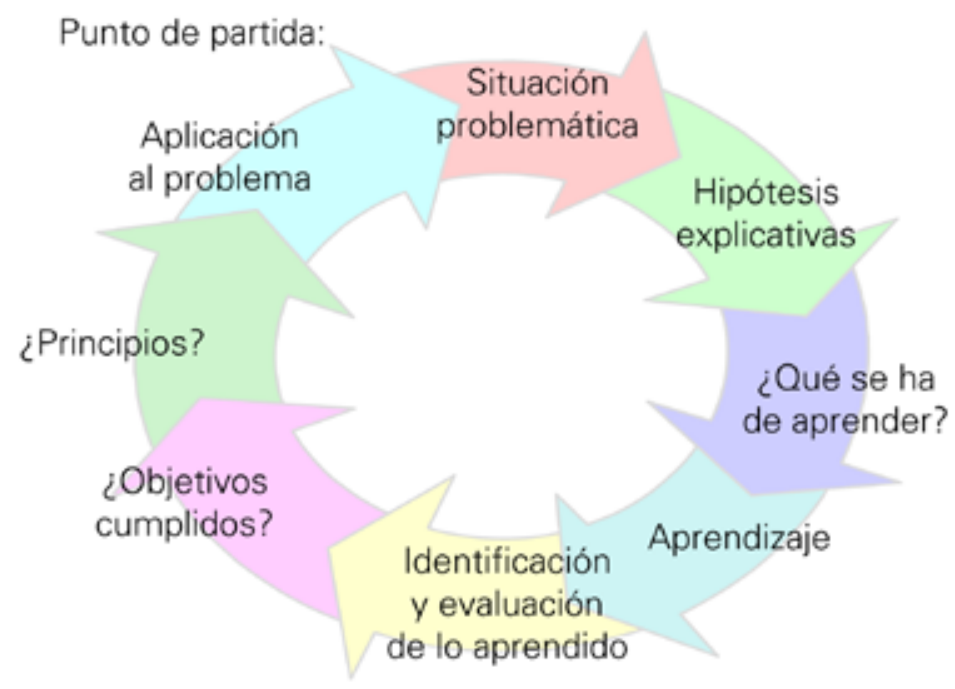

Figura 1. Ciclo de exploración de una situación problemática en el ABP.

Las definiciones de lo qué es el $\mathrm{ABP}$ son variadas, lo que conduce muchas veces a confusiones. Algunas de éstas incluyen la resolución de problemas. En un libro reciente, uno de los pioneros en el desarrollo de esta estrategia, Howard Barrows, incluye la resolución de problemas en lo que el título del libro ha denominado aPBL (authenticPBL) [3]. Sin embargo, la resolución de problemas es una extensión del ABP y puede $\mathrm{o}$ no incluirse en este método de aprendizaje. $\mathrm{Al}$ comienzo de la formación de un estudiante en un programa de $\mathrm{ABP}$ no debe esperarse que sea capaz de resolver problemas, ya que su base de conocimiento no es suficiente para hacer esto. Las situaciones que se le presentan van dirigidas a adquirir conocimientos, y no se espera que los posibles problemas que éstas contengan sean resueltos. Asimismo, con el progreso del estudiante en un programa, particularmente en aquellos de naturaleza profesional, como es la medicina, se espera que el estudiante sepa intervenir y trate de resolver los problemas presentados.

Los objetivos y las tareas que se deben cumplir en el ABP son:

- Utilizar estrategias de razonamiento para combinar y sintetizar la información proporcionada por el problema o situación en una o más hipótesis explicativas.
- Identificar necesidades de aprendizaje.

- A partir de lo aprendido, identificar los principios que puedan aplicarse a otras situaciones/problemas.

Al finalizar el análisis del problema o situación, los estudiantes deben identificar qué han aprendido y han de tratar de contestar las preguntas siguientes:

- ¿Qué cosas nuevas hemos aprendido al trabajar con el problema?

- ¿Cómo se relaciona este nuevo aprendizaje con lo aprendido previamente?

- ¿Cómo se relaciona este aprendizaje con los objetivos de aprendizaje?

- ¿Qué principios se han discutido y cuáles hemos aprendido?

- ¿Qué de lo aprendido nos ayudará a entender diferentes problemas o situaciones en el futuro?

- ¿Qué áreas de aprendizaje se han identificado importantes para el problema pero no se han explorado?

La figura 1 ilustra los elementos fundamentales del ABP.

Cuando en el programa de aprendizaje se incluye como objetivo la resolución de problemas, el ciclo de exploración del ABP se modifica (Fig. 2). 


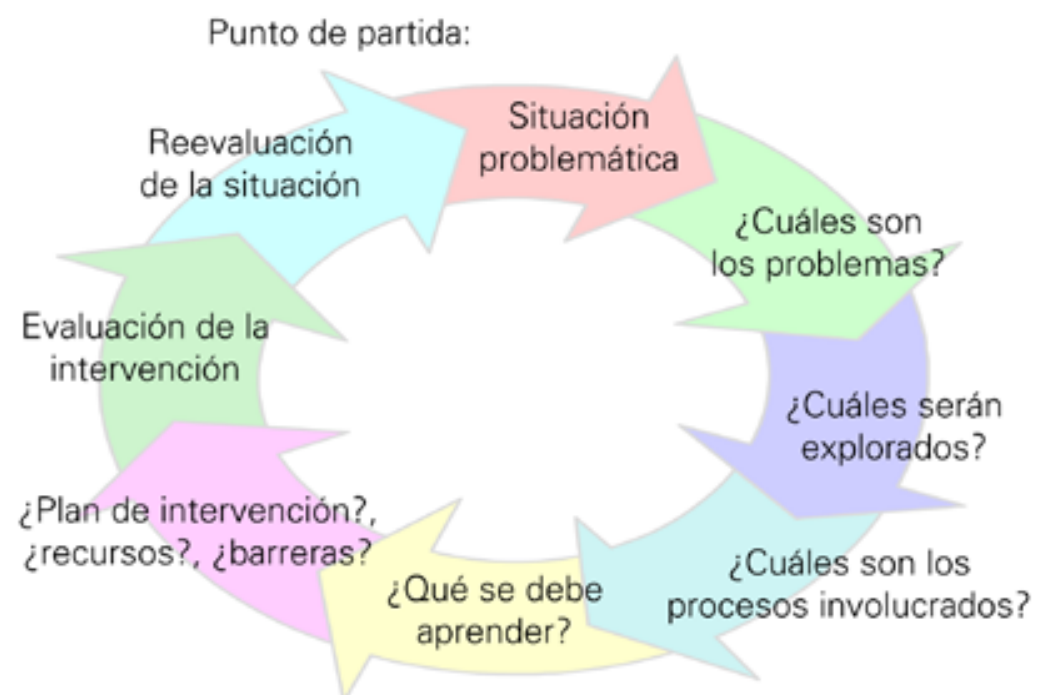

Figura 2. Ciclo de resolución de un problema en el ABP.

En ambos casos, con inclusión o no de la resolución del problema, el ABP se lleva a cabo dentro de un contexto determinado por:

- Los objetivos de aprendizaje (del programa y los objetivos individuales de los estudiantes).

- Los datos/información del problema o situación presentada.

- El conocimiento previo y las habilidades de cada estudiante.

\section{¿Qué se necesita para aplicar el ABP?}

\section{Objetivos de aprendizaje (resultados del aprendizaje)}

Los objetivos de aprendizaje (resultados del aprendizaje) son una descripción explícita, en términos concretos y sin ambigüedades, de lo que se espera que los estudiantes deban aprender o deban ser capaces de hacer como resultado del aprendizaje. Estos objetivos indican las circunstancias en las cuales se sabe que el estudiante ha adquirido las competencias establecidas por el programa (grado/asignatura/módulo o unidad de aprendizaje). El término 'competencia' en su acepción académica se ha definido como la combinación de atributos que describe cómo los estudiantes serán capaces de desenvolverse al finalizar el proceso educativo [4]. Dentro de las competencias se incluyen aquellas que se consideran genéricas (instrumentales, interpersonales y sistémicas) y las que son específicas de cada área temática.

Los objetivos de aprendizaje han de tener las características siguientes: han de ser relevantes a las competencias (pertinentes al perfil del titulado del programa), claros (sin ambigüedades), factibles (realizables en el tiempo acordado) y evaluables (establecen las únicas bases de la evaluación del estudiante).

En los objetivos de aprendizaje deben estar presentes los elementos siguientes :

- Las condiciones en las cuales se espera que el estudiante demuestre que ha cumplido los objetivos pertinentes.

- La actividad que el estudiante debe llevar a cabo para demostrar el cumplimiento del objetivo.

- El contenido (conocimiento/habilidad/actitud) que el estudiante ha de demostrar para ser considerado satisfactorio.

\section{Problemas/situaciones. ¿Qué es un 'problema' en el ABP?}

En la Universidad de McMaster se comenzó a aplicar el ABP para el aprendizaje de estudiantes 
que estaban al inicio de su programa de Medicina que no incluía la resolución de problemas [5]. Como en las fases posteriores del programa se utilizaron problemas clínicos, surgió la frase de 'aprendizaje basado en problemas' (problem based learning), que aparece en la mayoría de las publicaciones. Pero el ABP usualmente se aplica primero al uso de problemas (en el ABP el vocablo 'problema' podría considerarse con el sentido que le atribuye la primera definición del diccionario de la RAE: 'Cuestión que se trata de aclarar'), situaciones o escenarios que no requieren una solución. Las palabras utilizadas para referirse a lo que inicia el aprendizaje en el ABP han incluido problemas, situaciones problemáticas, escenarios y casos.

Lo importante en el ABP son las características del 'problema' que se utiliza. Se ha encontrado que un problema efectivo es aquel no estructurado, en el cual la información se presenta de forma progresiva y redactada de manera que propicia la discusión del grupo e incluye algunas veces frases de controversia. También se ha observado que se estimula la motivación del estudiante cuando la situación o el problema contienen elementos con los que éste puede identificarse $y$, fundamentalmente, que reflejan la realidad del futuro mundo laboral. El uso de palabras en la situación o el problema es deliberado y cuidadosamente elegido para estimular la identificación de áreas de aprendizaje, incluso su título. Se evitan datos superfluos y se describen los hechos sin hacer juicios o conclusiones, a menos que esto se incluya de forma deliberada para estimular la discusión.

El formato de los problemas es variado y depende de los objetivos de aprendizaje como de los recursos disponibles. El formato más común es el escrito, que permite a los estudiantes tener su propia copia del problema o situación y hacer notas en ésta, releerla y analizar cuidadosamente la información presentada. Otros formatos utilizados son vídeos, y en las ciencias de la salud, pacientes simulados y pacientes reales, particularmente en las etapas de resolución de problemas.

La selección de las situaciones o los problemas (casos) se basa exclusivamente en los objetivos de aprendizaje del programa. Ha sido de utilidad seguir los pasos siguientes:

1. Construir una lista de situaciones posibles según los objetivos de aprendizaje.
2. Adjudicar un valor educacional según su relevancia a esos objetivos.

3. Adjudicar la frecuencia con la cual la situación se encuentra en la realidad profesional.

4. Adjudicar el impacto que esa determinada situación pueda tener en el individuo o en el entorno profesional (comunidad, empresa, corporación).

5. Establecer un orden prioritario según los criterios anteriores.

6. Seleccionar los problemas a escribir.

En las tablas I, II y III se ilustran los resultados de los pasos 2, 3 y 4, respectivamente.

El establecimiento de las prioridades de selección de problemas que hay que preparar y la utilización de los tres criterios indicados en las tablas I, II y III es responsabilidad absoluta de los docentes y debe ser el resultado de un proceso de trabajo cooperativo y de consulta. Como se puede observar en la tabla I, cada problema/situación (caso), además de ofrecerle al estudiante la oportunidad de cumplir varios objetivos de aprendizaje, señala que para cada objetivo hay más de una oportunidad de llevarlo a cabo. Es decir, que no cumplir uno de los objetivos como resultado de la exploración de un caso puntual no significa que esto no se pueda llevar a cabo en el futuro con otro caso. Si bien esto permite cierta flexibilidad en el aprendizaje, requiere que los estudiantes desarrollen habilidades organizativas y de seguimiento para que no haya lagunas en el cumplimiento de los objetivos. El tutor facilitador puede jugar un papel importante en este proceso.

\section{Evaluación. ¿Cómo se evalúa en el ABP?}

Obviamente, esta es una de las áreas que presenta más dificultades desde que se pretende evaluar el conocimiento (adquisición, comprensión e utilización), las habilidades (tanto técnicas como de aprendizaje) y las actitudes (de gran relevancia hacia el comportamiento profesional y las relaciones interpersonales). En un sistema que pone énfasis en el aprendizaje autodirigido, los estudiantes han de conocer claramente cuáles son las pautas de evaluación, pautas que deben ser exclusivamente aquellas que se relacionan con los objetivos de aprendizaje del programa. Estos objetivos deben incluir ítems que permitirán al estudiante poder contestar satisfactoriamente preguntas de evaluación del conocimiento como, 
Tabla I. Relevancia de los problemas/casos de los objetivos de aprendizaje para determinar el valor educacional.

\begin{tabular}{ccccccc}
\hline & Caso A & Caso B & Caso C & Caso D & Caso E & Caso Z \\
\hline Objetivo 1 & Mucha & Poca & Mucha & Ninguna & Mucha & Mediana \\
\hline Objetivo 2 & Ninguna & Poca & Mucha & Mucha & Poca & Mucha \\
\hline Objetivo 3 & Mediana & Mucha & Mediana & Poca & Ninguna & Mucha \\
\hline Objetivo 4 & Ninguna & Mucha & Poca & Mucha & Mucha & Ninguna \\
\hline Objetivo 5 & Mucha & Mediana & Mucha & Mucha & Mediana & Poca \\
\hline Objetivo $n$ & Mediana & Poca & Mucha & Poca & Mucha & Ninguna \\
\hline
\end{tabular}

asimismo, demostrar: responsabilidad (p. ej., avisar en caso de ausencia, respetar los horarios, llevar a cabo la búsqueda de información a la que se han comprometido); habilidades de aprendizaje (p. ej., demostrar la capacidad de formular hipótesis explicativas de la situación, colaborar efectivamente para programar y llevar a cabo un plan de estudio, justificar la información recogida con la evidencia resultante de un análisis crítico y organizarse en la gestión del tiempo); habilidades de comunicación (p. ej., presentar la información de forma ordenada, expresarse clara $\mathrm{y}$ concisamente); habilidades interpersonales ( $\mathrm{p}$. ej., mostrar tolerancia con los colegas del grupo, esperar el momento apropiado para intervenir y saber escuchar).

Se han descrito varios métodos para la evaluación individual de cada estudiante que le permitirían demostrar ser competente en haber cumplido los objetivos de aprendizaje que el programa ha establecido. En varias publicaciones se encontrarán descripciones más detalladas de la evaluación en programas que utilizan el ABP [6-8].

\section{Evaluación basada en observaciones recogidas en las sesiones de tutoría} Este aspecto de la evaluación, por su naturaleza esencialmente formativa, se ha considerado de importancia primordial en los programas que utilizan el ABP. Los elementos que forman parte de esta evaluación son la autoevaluación, la coevaluación y la evaluación por el tutor facilitador. Esta evaluación se basa en lo observado en las sesiones de tutoría y no en juicios de las características personales de los integrantes del grupo. La descripción de lo observado permite identificar fortalezas y áreas que se deben corregir y no debe incluir ninguna especulación de las razones que motivaron ese comportamiento. Este proceso de evaluación se lleva a cabo de forma abierta, en un tiempo específico asignado en una sesión de tutoría y siguiendo pautas o criterios previamente establecidos.

En las sesiones iniciales de tutoría, tanto la autoevaluación como la coevaluación son procesos incómodos y el grupo necesita desarrollar un grado alto de confianza para poder hacerla efectivamente. El tutor facilitador ayuda en esta tarea, de manera que es el que inicia la autoevaluación y ratifica, aclara o discrepa de la evaluación que hacen los estudiantes de ellos mismos o de sus compañeros.

\section{Evaluación sumativa}

La evaluación individual permite observar si el estudiante, de forma no dependiente del grupo de tutoría, es competente en: 
Tabla II. Prevalencia de los problemas/casos como factor para determinar la selección.

\begin{tabular}{lllllll}
\hline Caso A & Caso B & Caso C & Caso D & Caso E & $\ldots$ & Caso Z \\
\hline Mucha & Poca & Mucha & Ninguna & Mucha & & Mediana \\
\hline
\end{tabular}

Tabla III. Impacto ${ }^{\text {a }}$ de los problemas/casos como factor para determinar la selección.

\begin{tabular}{|c|c|c|c|c|c|c|}
\hline Caso A & Caso B & Caso C & Caso D & Caso E & $\ldots$ & Caso Z \\
\hline Mucho & Poco & Mucho & Ninguno & Mucho & & Mediano \\
\hline \multicolumn{7}{|c|}{$\begin{array}{l}\text { aEl impacto es una variable de carácter subjetivo que puede cambiar de tiempo en tiempo en función del problema. Unos ejemplos podrían } \\
\text { ser una situación problemática basada en la crisis inmobiliaria o los efectos en la salud de una comunidad de problemas debidos a la incle- } \\
\text { mencia del tiempo. }\end{array}$} \\
\hline
\end{tabular}

- Identificar áreas de conocimiento relevantes para los objetivos de aprendizaje y la situación problemática presentada.

- Indicar el conocimiento previo que ha llevado a la selección de estas áreas.

- Seleccionar de estas áreas aquellas que considera de mayor importancia en relación con la situación problemática y dar una justificación del porqué de la selección.

- Llevar a cabo una búsqueda efectiva de información relevante a las áreas seleccionadas.

- Analizar críticamente la información recogida y convertirla en conocimiento que sea relevante a la situación problemática.

- Identificar entre este conocimiento los principios aplicables a otras situaciones problemáticas.

- Demostrar comprensión y validez del conocimiento adquirido.

- Aplicar el conocimiento adquirido durante este ejercicio de evaluación como el conocimiento previo a la situación problemática.

Se han desarrollado varios instrumentos para llevar a cabo esta evaluación que trata de ser lo más continuada posible, y se ha evitado el 'examen final' que da información sobre los resultados del aprendizaje sin una oportunidad adecuada para realizar cambios o correcciones. En la bibliografía se pueden encontrar la descripción de algunos de estos instrumentos de evaluación $[9,10,11]$.

\section{¿Cuáles son las etapas más comunes en el ABP?}

\section{Lluvia de ideas y plan de aprendizaje}

Una vez presentada la situación problemática, lo más frecuente es que los estudiantes discutan y hagan una lista de las áreas de conocimiento (temas) que consideran relevantes a la situación. En esta etapa cualquier intervención es válida y se debe prestar atención a que no se filtre ninguna opinión; todo es apropiado para un análisis posterior. De esta lista, usualmente muy extensa, resultado de lo que se podría llamar una 'tempestad o lluvia de ideas', se construye un plan de estudio o plan de aprendizaje que contiene objetivos específicos para la situación problemática que se discute. Estos objetivos, identificados por el grupo de estudiantes, generalmente están relacionados con los objetivos de aprendizaje determinados por el programa (asignatura, módulo 
o programa de aprendizaje), pero puede incluir algunos que son adicionales. Cada grupo debe acordar el plan de estudio o aprendizaje para cada problema. El grupo debe analizar el plan de aprendizaje y tener en cuenta que debe focalizarse en su mayor parte -no necesariamente de forma exclusiva- en las áreas que sean más relevantes para llevar a cabo los objetivos del programa. Pero este plan de aprendizaje puede incluir objetivos individuales y del grupo no contemplados por los objetivos de aprendizaje programáticos. Acordar un plan de aprendizaje permite la expresión de los intereses individuales -producto de diferentes experiencias, fortalezas y debilidades-, así como del grupo. Los objetivos de aprendizaje programáticos a menudo sirven como guía para elaborar el plan de aprendizaje o plan de estudio.

Una vez que los temas que forman el plan de aprendizaje se han identificado, reorganizado y clarificado, los miembros del grupo eligen el tema o temas en los que desean trabajar.

No es aconsejable que se escojan todos los temas de búsqueda; es probable que esto haga que la investigación resulte superficial (fundamentalmente por falta de tiempo). Es esencial que se identifique el conocimiento previo de los estudiantes sobre los temas que surgieron en la lluvia de ideas y en el plan de aprendizaje. Esto permite una elección de temas que incrementan el conocimiento de los estudiantes y no de aquellos temas que ya conocen.

Una vez identificados los temas de aprendizaje, es aconsejable que el próximo paso sea reconocer las fuentes previsibles de información del conocimiento. Hay un amplio abanico de posibilidades que va desde los libros de texto y las monografías hasta docentes y expertos tanto en el ámbito académico como en la comunidad; se espera que cada miembro del grupo contribuya con sugerencias. El tutor facilitador docente puede ser de ayuda aclarando dudas sobre la validez de algunos recursos identificados por los estudiantes (en caso de que el docente tenga algún conocimiento de esas fuentes de información).

\section{Seguimiento del problema o de la situación}

En el segundo encuentro o segunda sesión de la tutoría, lo más frecuente es que el grupo discuta las fuentes y los recursos utilizados en el período del estudio. Cada miembro resume brevemente las fuentes que ha explorado, el porqué de su selección y los problemas encontrados en la búsqueda. Ésta es una oportunidad para que el grupo comparta las fuentes de información y para aprender cómo acceder a éstas, y evaluarlas mediante un análisis crítico. Desde el principio, el grupo debe debatir cómo evaluar críticamente la información recogida (p. ej., la reputación de los autores, los métodos de búsqueda empleados y los métodos estadísticos aplicados). Este proceso es parte de las habilidades que el futuro profesional requerirá a lo largo de toda su carrera.

En esta segunda sesión todos los miembros del grupo deben tener una oportunidad para aplicar la información que han estudiado al problema que se discute. De esta manera, se facilita que el estudiante se esté capacitando para la evaluación crítica y la corrección de su conocimiento previo en función del nuevo conocimiento adquirido, y que desarrolle la habilidad de evaluar críticamente su análisis inicial del problema.

La aportación de información que cada estudiante realiza puede complementarse con artículos, diagramas y otro material. Pero este proceso no constituye una presentación de la información recogida sino una reconstrucción e interpretación del conocimiento adquirido. De aquello que han aprendido en relación con el problema particular, los estudiantes deben extraer los principios y los conceptos que pueden ser aplicados a otros problemas.

El problema debe ser reevaluado con la revisión del plan de aprendizaje y/o la lista de hipótesis, indicar qué cambios deberían hacerse y qué hipótesis deben ser ratificadas, alteradas o cambiadas. Eso permite al estudiante y al grupo identificar temas que no se han tratado con profundidad; estos temas pueden formar parte de un plan de estudio posterior. La información obtenida mediante el aprendizaje autodirigido es aplicada nuevamente al problema de forma activa y estimulante, lo cual aumenta su comprensión y hace que la nueva información sea integrada al conocimiento básico de cada estudiante. El conocimiento discutido también permite generar nuevas preguntas e ítems que pueden establecer nuevos planes de aprendizaje.

\section{Resumen del conocimiento y abstracción}

Al finalizar el análisis de la situación problemática, los estudiantes deben identificar qué han 
aprendido y tratar de contestarse las preguntas siguientes:

- ¿Qué se ha aprendido de nuevo trabajando con el problema?

- ¿Cómo se relaciona este aprendizaje con los objetivos de aprendizaje?

- ¿Qué principios nuevos se han discutido y cuáles se han aprendido?

- ¿Qué de lo aprendido ayudará a entender diferentes problemas en el futuro?

- ¿Qué áreas de aprendizaje se han identificado pero no se han explorado?

\section{¿Cuál es la evolución natural de un grupo de tutoría?}

Se han descrito varios procesos evolutivos del grupo de tutoría que utiliza el ABP. El cuaderno de notas de un estudiante permite obtener una idea de las distintas etapas en este proceso evolutivo.

Etapa 1. ¿Qué se supone que se debe hacer? 'Después de las primeras tutorías no estamos seguros de lo que debemos hacer. La confusión es total. Nos movemos sin estar seguros de a qué lugar debemos ir. Nos sentimos como si tratásemos de completar un rompecabezas sin tener la foto del producto final. Me siento como en un callejón oscuro con poca luz y teniendo que seguir recorriéndolo sin saber lo que me espera en la salida. Pero me dije, ¡no flipes, tío!’

Etapa 2. ¿Se aprende lo suficiente?

'Aprendimos que hacer presentaciones de lo que estudiamos era aburrido y no aprendíamos mucho desde que estábamos pendientes de cuándo llegaría nuestro turno para mostrar lo que preparamos. Nos dimos cuenta de que después de una introducción de cinco minutos, el compartir las fuentes de información era suficiente para que cada uno estudiara los temas. Sentimos que el ritmo es demasiado lento y que no utilizamos bien el tiempo. No somos eficientes. Nos ayudaría a ahorrar tiempo si la tutora nos dijera claramente cuál es el camino correcto.'

\section{Etapa 3. ¿Se aprende algo útil?}

'Algunos días nos preguntábamos si después de tanto trabajo estábamos aprendiendo algo útil. Pero hoy hemos observado los objetivos de aprendizaje para ver cuánto llevamos a cabo, y nos he- mos sorprendido de lo mucho que hemos aprendido. Nos hemos dado cuenta de que llegar a una meta requiere un trabajo duro, pero hemos demostrado que tenemos las habilidades necesarias para aprender y que nuestro esfuerzo individual nos hará llegar donde nos propongamos y cumpliremos con las tareas asignadas. Nos sentimos más seguros y con menos ansiedad.'

Etapa 4. ¿Se ha estabilizado el proceso de aprendizaje?

'Se dice que usar metáforas expresa realmente lo que sentimos. Tras varias tutorías, nos sentimos como árboles jóvenes en un bosque. Hemos crecido y tenemos ramas y hojas nuevas y nos sentimos con mucha confianza en lo que hemos aprendido. Tratamos de tener más sol para sentirnos más fuertes. Los profesores son como árboles más grandes que quizás sean más sabios que nosotros, aunque a veces nos tapan el sol, pero también aprendemos de ellos. Siento que los profesores son como jardineros que vienen de vez en cuando y nos dan fertilizante. Pero también nos podan para hacernos más fuertes y crecer mejor. Esto a veces es doloroso, pero el resultado muestra que ha sido necesario y que nos ha ayudado a crecer más fuertes. A pesar de las noches en vela, la frustración cuando las cosas salen mal, los momentos de enfado, recuerdo los momentos de júbilo, el nuevo aprendizaje y las nuevas experiencias. Pienso que seguiré disfrutando del yin yang que es el ABP, trabajo duro pero placentero. Veo el futuro muy prometedor, y en aquellos días en que las cosas no salgan bien pensaré que, como dijo Scarlett O'Hara en Lo que el viento se llevó, «mañana será otro día».

\section{¿Se puede usar el ABP en grupos grandes?}

La implementación del ABP en la mayoría de los casos se lleva a cabo en grupos pequeños, como se concibió en su formato original. Sin embargo, limitaciones fundamentalmente de recursos tanto humanos como físicos han llevado a implementar el ABP en grupos grandes. Una secuencia de actividades puede ser la siguiente:

\section{Primera sesión}

- Paso 1. Presentación del problema a la clase, generalmente por escrito (10 minutos). 
- Paso 2. Trabajo en grupos pequeños con las instrucciones de discutir el problema e identificar objetivos de aprendizaje de interés para el grupo (20 minutos).

- Paso 3. Informe de cada grupo al resto de la clase por un representante de cada grupo (20 minutos).

- Paso 4. Selección de temas de estudio independiente o contribución del docente (10 minutos). Éste recomienda aquellos temas que considera que deben ser de estudio individual y aquellos en los que un profesor dará una clase, no expositiva pero conceptual.

\section{Segunda sesión}

Clase del docente que incluye tiempo para preguntas y aclaraciones.

\section{Tercera sesión}

- Paso 1. Trabajo en grupos (30 minutos). Los estudiantes discuten dentro del grupo lo aprendido e identifican áreas de dudas o nuevos temas que deben aprender relacionados con los objetivos de aprendizaje y relevantes al problema.

- Paso 2. Informe de cada grupo al resto de la clase sobre los temas que necesitan aclaraciones y posibles nuevos temas que se pueden explorar (10 minutos).

- Paso 3. Clarificaciones y selección de áreas de estudio individual o presentaciones por el docente (20 minutos).

\section{Cuarta sesión}

Clase del docente sobre el tema acordado.

\section{Quinta sesión}

- Paso 1. Aclaraciones de dudas.

- Paso 2. Revisión de objetivos de aprendizaje cumplidos.

Un nuevo problema representa una secuencia similar.

\section{El rol del tutor facilitador}

El docente es clave para facilitar el aprendizaje autodirigido. En el trabajo en grupos pequeños, particularmente en el estudio de problemas que son interdisciplinarios, el rol del docente es fundamentalmente el de facilitador del aprendizaje del estudiante. Hay que asegurar que los estudiantes tengan claro el rol del tutor o facilitador: estimular el debate. Los tutores facilitadores no son las fuentes de información sobre el problema, ni siquiera cuando tienen conocimiento de algunos aspectos del problema. El tutor facilitador no enseña en el sentido convencional de la palabra, sino que facilita el proceso de aprendizaje mediante preguntas que pretenden provocar el pensamiento y el debate entre los estudiantes. El tutor facilitador debe desafiar el pensamiento y las ideas de los estudiantes siempre que tenga dudas de si éstos entienden lo que están discutiendo. La pregunta más común de un tutor es ‘por qué?’ Los estudiantes deben cuestionarse a sí mismos y no depender del tutor facilitador para este desafío; a través de estos cuestionamientos analizan y discuten la información y el conocimiento relacionado con el problema y los principios que ese conocimiento ilustra.

El rol del tutor como facilitador es distinto del comúnmente atribuido al tutor en muchos programas de aprendizaje. Ése se puede definir como un docente que posee habilidades en la facilitación del aprendizaje y que promueve el pensamiento crítico, el funcionamiento eficiente y eficaz del grupo, el aprendizaje individual, la evaluación en las tutorías y el aprendizaje centrado en el estudiante, es decir, que los estudiantes son principalmente responsables de su propio aprendizaje.

Además, el tutor facilitador comprende los objetivos generales y de aprendizaje del programa educativo y asegura que los estudiantes ven la relevancia de los ítems generados por el problema, posee conocimiento de los recursos de aprendizaje disponibles para los estudiantes y sirve de modelo como profesional responsable.

\section{Las intervenciones del tutor}

Basado en un modelo de análisis de las intervenciones de los tutores facilitadores, se ha desarrollado un cuestionario de autoevaluación del tutor (CAET) con el cual el facilitador puede examinar sus acciones en diferentes situaciones que surgen en las sesiones de tutoría. El modelo ilustra seis categorías de intervenciones, dos tipos de orientación de la acción y cuatro modalidades de facilitación. La figura 3 ilustra este modelo. 


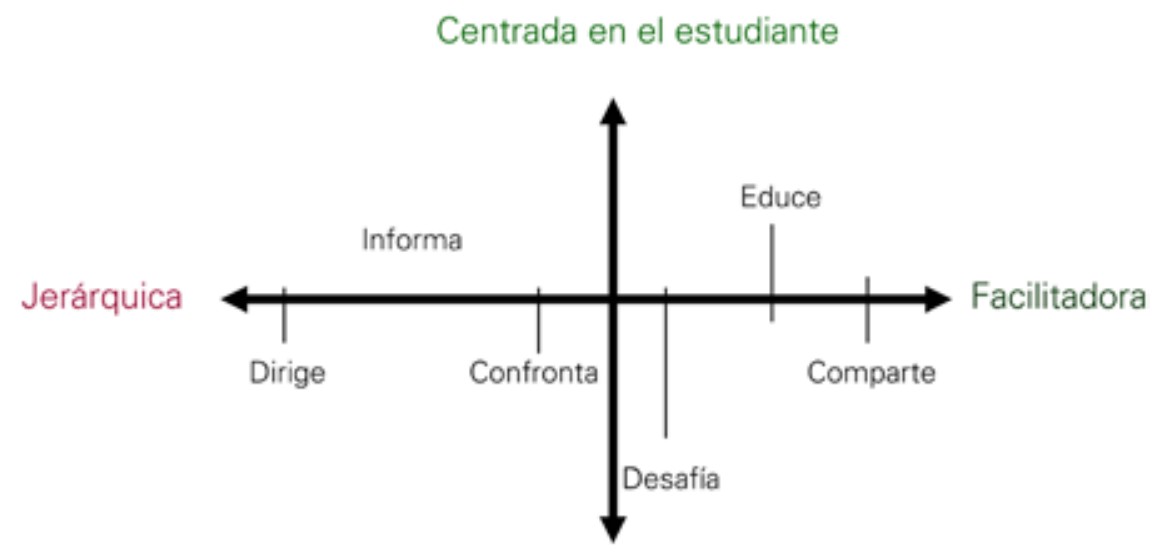

Centrada en el tutor

Figura 3. Ejes de continuidad en las intervenciones del tutor facilitador.

Los resultados del análisis de más de 200 CAET correspondientes a 5.251 respuestas muestran que la mayoría de los docentes $(61,8 \%)$ elige el cuadrante que corresponde a la intervención facilitadora centrada en el estudiante. Sólo una minoría $(6,1 \%)$ se ubica en el cuadrante de la intervención jerárquica centrada en el tutor.

\section{Reflexiones finales}

La bibliografía es pletórica en los estudios comparativos del ABP y el currículo convencional, y no es el propósito de este artículo hacer una revisión de los resultados. Recientemente, Koh et al han publicado los resultados de un metaanálisis extenso en el que comparan el efecto del ABP y del currículo convencional en el desarrollo de competencias profesionales después de la titulación [12].

\section{El aprendizaje autodirigido}

Se ha considerado que lo esencial del ABP es el aprendizaje autodirigido en el cual el estudiante aprende de forma autónoma. Eso significa un cambio fundamental en el rol del docente que debe facilitar este proceso. Sin embargo, lo que a me- nudo se entiende por aprendizaje centrado en el estudiante (a menudo referido como 'autoaprendizaje'), en la praxis no es siempre congruente con el aprendizaje autodirigido. La bibliografía sobre la relación entre el aprendizaje autodirigido y el aprendizaje autorregulado no consiste en considerar cuál incluye cuál, aunque la revisión hecha recientemente por Loyens y colaboradores parece concluir que el primero es más amplio que el segundo [13]. Esta revisión de la bibliografía indica que si bien la mayoría de los estudios señala resultados positivos en el efecto que el ABP tiene sobre el aprendizaje autodirigido, también indica que lo que esto significa para ambos, estudiantes y docentes, desempeña un papel importante en la interpretación de los resultados.

En la conocida leyenda griega Dédalo construye alas fijadas al cuerpo con cera para que él y su hijo, Ícaro, puedan escapar de la prisión en que los confinó el rey Minos. A pesar de la advertencia de no volar demasiado alto porque el calor del sol derretiría la cera, sabemos que Ícaro, intoxicado por la libertad adquirida, voló tan alto que el ardiente sol ablandó la cera y desprendió las alas resultando en la caída y muerte de éste. El ABP da al estudiante la responsabilidad de autorregular su aprendizaje, pero debemos ser precavidos para evitar el desastre que puede ocurrir en un uso incontrolado de esta responsabilidad. 


\section{Bibliografía}

1. Araújo UF, Sastre GS, eds. El aprendizaje basado en problemas. Una nueva perspectiva de la enseñanza en la universidad. Barcelona: Gedisa; 2008.

2. Araújo UF, Sastre GS, eds. Aprendizagem baseada em problemas no ensino superior. São Paulo: Summusl; 2009.

3. Barrows HS, Wee Keng Neo L. Principles and practice of Apel. Singapore: Pearson Prentice Hall; 2007.

4. Proyecto Tuning. Informe final. Bilbao: Universidad de Deusto; 2003.

5. Branda LA. El aprendizaje basado en problemas. El resplandor tan brillante de otros tiempos. In Araújo UF, Sastre G, eds. El aprendizaje basado en problemas. Una nueva perspectiva de la enseñanza en la universidad. Barcelona: Gedisa; 2008. p. 17-46.

6. Cònsul-Giribet M, ed. Historia de un cambio. Un currículo integrado con el aprendizaje basado en problemas. Escola Universitària d'Infermeria Vall d'Hebron. Barcelona: Enciclopèdia; 2007. URL: http://www.vhebron.es/do/eui/ publicacions.htm. [20.02.2009].

7. Del Valle M, García-Diéguez M, Moscoso N, Rotstein N,
Silberman P. Guía de evaluación del ciclo inicial de la carrera de Medicina. Bahía Blanca: Universidad Nacional del Sur; 2007. p. 105-45.

8. Bermejo F, Pedraja MJ. La evaluación de competencias en el ABP y el papel del portafolio. In García-Sevilla J, ed. El aprendizaje basado en problemas en la enseñanza universitaria. Murcia: Universidad de Murcia; 2008. p. 91-111.

9. Woods DR. Preparing for PBL. 2006. URL: http://www. chemeng.mcmaster.ca/innov1.htm. [20.02.2009].

10. Font A, ed. Proyecto CarpeTiki. El uso del portafolio electrónico en entornos semipresenciales y de aprendizaje por problemas. URL: http://gclub.ub.es/carpetiki/tiki-index. php. [20.02.2009].

11. Kustra E. Using Problem Based Learning for Assessment in Large Classes:Triple-Jump. URL: http://resources. glos.ac.uk/ceal/resources/casestudiesactivelearning/ activelearningcasestudies/casestudy7.cfm [20.02.2009].

12. Koh GCH, Khoo HE, Wong ML, Koh D. The effects of problem-based learning during medical school on physician competency: a systematic review. CMAJ 2008; 178: 34-41.

13. Loyens SMM, Magda J, Rikers RJMP. Self-directed learning in problem-based learning and its relationship with self-regulated learning. Educ Psychol Rev 2008; 20: 441-27. 